\title{
Exacerbation of Crohn's disease after insertion of a levonorgestrel intrauterine system: a case report
}

\author{
Jacqueline Wakeman, MRCGP, DFFP, General Practitioner, Langton Medical Group, St Chad Health Centre, Lichfield, UK \\ Correspondence: Dr J G Wakeman, Langton Medical Group, St Chad Health Centre, Dimbles Lane, Lichfield WS13 7HT, UK \\ (Accepted 12th May 2003) \\ Journal of Family Planning and Reproductive Health Care 2003; 29(3): 154
}

\begin{abstract}
The case report describes a relapse of Crohn's disease in a woman soon after the fitting of a levonorgestrel intrauterine system (IUS). Whilst the published evidence on the effects of levonorgestrel IUS in patients with pre-existing inflammatory bowel disease is limited, reports to date suggest that caution should be exercised when considering fitting an IUS in such women, even if there appears to be much to gain from an expected reduction in menorrhagia.
\end{abstract}

\section{Case study}

A 38-year-old woman with Crohn's disease (CD) was referred by a colleague in general practice for consideration of insertion of a levonorgestrel intrauterine system (IUS) as her periods had become increasingly heavy despite the use of tranexamic acid. CD had been diagnosed at the age of 21 years and she had had an ileo-colectomy 12 years previously, subsequently followed by a cholecystectomy and operations for adhesiolysis. The combined oral contraceptive pill had been used both before and after diagnosis of $\mathrm{CD}$ and she had a flare of her CD following her first pregnancy. However, her $\mathrm{CD}$ had remained well controlled on a combination of mesalazine and loperamide for at least 12 months prior to the request for an IUS.

An intrauterine device would not generally be the first choice for contraception in a patient with inflammatory bowel disease (IBD) because of the difficulty of differentiating between a flare-up of IBD and pelvic inflammatory disease. ${ }^{1}$ In view of the fact that this particular patient had much to gain from an IUS in terms of control of menorrhagia, and the fact that her CD was well controlled, an IUS was fitted. High-vaginal and chlamydia swabs were taken prior to fitting and were negative.

At a 6-week check the patient reported that, since the IUS had been fitted, in addition to the expected irregular vaginal bleeding, she had been experiencing ankle swelling in the evenings. Also, her CD had flared up in the 2 weeks following fitting but had settled at the time of review.

Some 14.5 weeks after IUS insertion the patient returned requesting removal of the device as her $\mathrm{CD}$ was getting much worse, as was the ankle swelling. On examination she had obvious pitting oedema over the right ankle and slight oedema over the left. There were no symptoms suggestive of pelvic sepsis and D-dimer was negative. The IUS was removed as the patient felt the exacerbation in her CD was causally related to the IUS.

Following IUS removal the ankle swelling settled within a couple of weeks and the CD symptoms settled more gradually on usual medication over the following 3 months. The patient had not noted ankle swelling with any previous flare-ups in CD.

A yellow card was submitted to the Committee on Safety of Medicines (CSM) West Midlands.

\section{Discussion}

The 5-year report on clinical performance of the levonorgestrel IUS in a cohort of 678 women, by Cox et $\mathrm{al}^{2}$ prompted this case report. In the section reporting 'serious adverse events', it was noted that a 33-year-old woman with a history of $C D$ had been hospitalised with septicaemia 5 days following IUS fitting, though whether the septicaemia was due to the IUS fitting or the CD was debatable. Another woman had an exacerbation of ulcerative colitis (UC) and acne rosacea 25 months after IUS fitting. It is not clear from the report whether these two patients represented the total of all patients in the cohort with pre-existing IBD. However, since the prevalence of $\mathrm{CD}$ is in the region of 145 per 100000 , and that of UC 244 per 100000 , this is quite possible. ${ }^{3}$

$\mathrm{UC}$ and $\mathrm{CD}$ are both chronic relapsing disorders. It is not unexpected, therefore, that exacerbations of IBD will occur over time whilst a patient has an IUS in situ, whether or not the device has any effect on the underlying disease process. The practical difficulty will be determining whether abdominal symptoms, particularly if only mild to moderate, are IUS-induced (and should prompt IUS removal) or a part of the natural history of the underlying bowel disorder. A MEDLINE search revealed no references relating specifically to the use of IUS in patients with IBD. In addition, the manufacturers of Mirena ${ }^{\circledR}$ have received no reports of adverse effects from IUS use in patients with CD (Schering Health Care Ltd, personal communication).

As with the report by $\mathrm{Cox},{ }^{2}$ the onset of bowel symptoms after IUS insertion in this patient with previously well-controlled CD gives cause for concern. It was difficult to ascertain whether the physical presence of the device precipitated symptoms, whether it was due to a change in the local hormonal environment, or indeed whether it was coincidental.

There is some evidence that bowel habits are affected by the hormonal environment in the premenstrual and menstrual phase of a cycle, particularly in patients with CD. ${ }^{1}$ Whilst current or previous use of combined oral contraception is thought by some to predispose to exacerbations of $\mathrm{CD},{ }^{4}$ others, however, disagree ${ }^{5}$ (see also CEU Guidance document, page 132).

Clinicians with experience of IUS use in patients with IBD are encouraged to share their experiences so that a more informed picture use can be gained.

Statements on funding and competing interests

Funding. None identified.

Competing interests. None identified.

References

1 Kane S. Caring for women with inflammatory bowel disease. $J$ Gend Specif Med 2001; 4: 54-59.

2 Cox M, Tripp J, Blacksell S. Clinical performance of the levonorgestrel intrauterine system in routine use by the UK Family Planning and Reproductive Health Research Network: 5-year report. J Fam Plann Reprod Health Care 2002; 28: 73-77.

3 Rubin GP, Hungin APS, Kelly PJ, et al. Inflammatory bowel disease: epidemiology and management in an English general practice population. Aliment Pharmacol Ther 2000; 14: 1553-1559.

4 Clinical Alert. Smoking, use of oral contraceptives, and medical induction of remission were risk factors for relapse in Crohn's disease. Gut 1999; 44: 311-312.

5 Cosnes J, Carbonnel F, Carrat F, et al. Oral contraceptive use and the clinical course of Crohn's disease: a prospective cohort study. Gut $1999 ;$ 45(2): 218-222. 